\title{
Le site mésolithique de Fumichon «Vallée de la Pâquine » (Calvados)
}

The Mesolithic Site of Fumichon "Vallée de la Pâquine" (Normandy, France)

Emmanuel Ghesquière, Patrick Auguste et Jean-Luc Piel-Desruisseaux

\section{OpenEdition}

\section{Journals}

Édition électronique

URL : https://journals.openedition.org/rao/1704

DOI : $10.4000 /$ rao. 1704

ISBN : 978-2-7535-2790-4

ISSN : $1775-3732$

\section{Éditeur}

Presses universitaires de Rennes

\section{Édition imprimée}

Date de publication : 30 décembre 2012

Pagination : $7-24$

ISBN : 978-2-7535-2641-9

ISSN : 0767-709X

\section{Référence électronique}

Emmanuel Ghesquière, Patrick Auguste et Jean-Luc Piel-Desruisseaux, «Le site mésolithique de Fumichon «Vallée de la Pâquine » (Calvados) », Revue archéologique de l'Ouest [En ligne], 29 | 2012, mis en ligne le 30 décembre 2014, consulté le 22 août 2022. URL : http://journals.openedition.org/rao/ 1704 ; DOI : https://doi.org/10.4000/rao.1704

Ce document a été généré automatiquement le 22 août 2022.

Tous droits réservés 


\section{Le site mésolithique de Fumichon "Vallée de la Pâquine » (Calvados)}

The Mesolithic Site of Fumichon "Vallée de la Pâquine" (Normandy, France)

Emmanuel Ghesquière, Patrick Auguste et Jean-Luc Piel-Desruisseaux

1 La série de Fumichon est issue dans un premier temps d'un ramassage au sein d'un éboulis par l'un d'entre nous (Jean-Luc Piel-Desruisseaux), en distinguant trois niveaux qui n'ont pas révélé de différence en ce qui concerne les décomptes de mobilier (fig. 1), puis d'un sondage dans les lambeaux de couche plus ou moins en place (Jean-Luc PielDesruisseaux et Dominique Cliquet, SRA). Apparemment homogène, la série comprend 1482 artefacts d'une longueur supérieure à $1,5 \mathrm{~cm}$, parmi lesquels 114 outils du fonds commun et 26 armatures (plus 5 ébauches probables) ainsi que 473 esquilles. 
Figure 1 : Localisation géographique et topographique. Figure 1: geographical and topographic Localization.

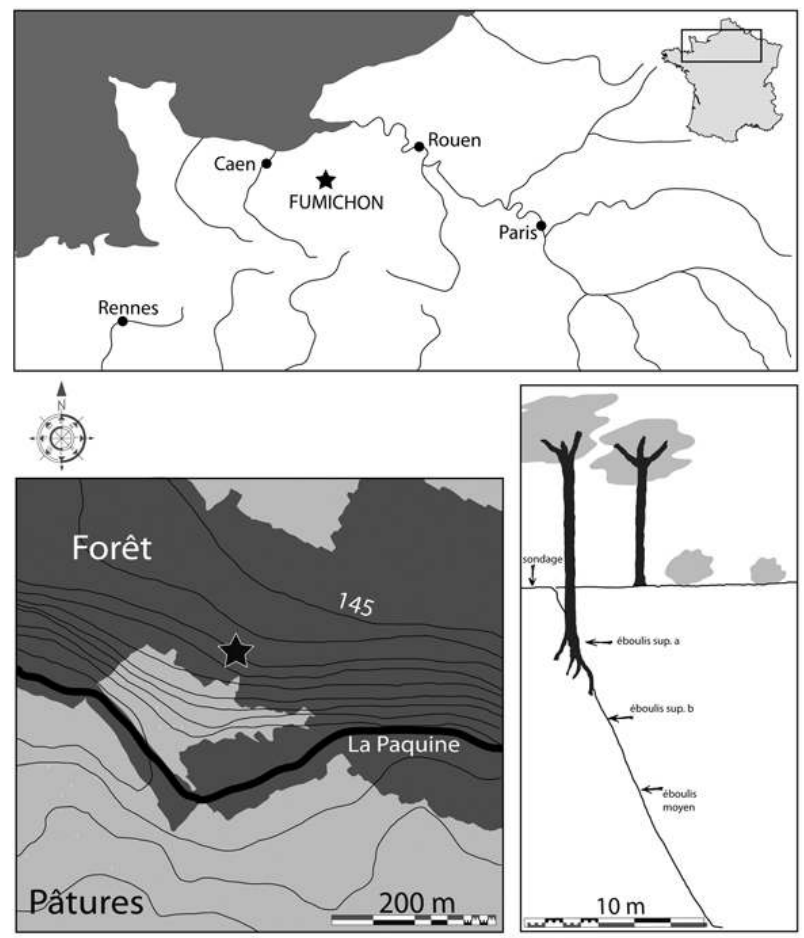

\section{Localisation topographique et méthode d'intervention}

2 Le site est installé sur un petit plateau surmontant d'une trentaine de mètres la vallée de la Pâquine, modeste affluent de la Touques (fig.1). Les flancs du coteau ont été utilisés comme carrières d'extraction de craie (amendement). C'est dans les éboulis couvrant le haut du front de carrière qu'ont été identifiés les premiers indices, puis qu'ont eu lieu les premières campagnes de ramassage. Il s'agit de produits de débitage, nucléus, outils, armatures contenus dans une argile et, dans le bas de l'éboulis, de quelques fragments osseux inclus dans une zone de poudre crayeuse.

Dans un premier temps a été effectué un prélèvement de matériel dans la partie ouest de l'éboulis sur une hauteur de dix mètres à partir du sommet. Puis, dans un second temps un sondage sur la bordure du plateau, surplombant l'éboulis, a été réalisé. Il couvre huit mètres carrés, découpé en parcelles nommées "ouest», "est» et " extrême est ». La surface du sol est couverte de rognons de silex au cortex altéré et d'un peu d'humus traversé par de nombreuses racines d'arbres. Plus profondément, une couche d'argile jaune contient de nombreux rognons de silex branchus au cortex tendre. L'épaisseur de cette couche d'argile varie de quelques centimètres à une vingtaine de centimètres dans des sortes de rigoles, avant de devenir très compacte et homogène. Le matériel lithique, identique à celui de l'éboulis, est dispersé de la surface à la couche d'argile d'aspect pur. Aucun reste osseux n'a été trouvé dans les sondages.

Aucun repérage positionnel précis des pièces n'a été fait en raison de l'impression d'un «brassage » du matériel. Il n'a pas été réalisé de tamisage à cause de la consistance de 
la couche argileuse, difficile à défloquer. Cela contribue à expliquer le déficit en pièces de petite taille, comme les esquilles et les armatures.

\section{Interprétation taphonomique et homogénéité de la série}

5 Le mobilier n'est plus en place sur le site. Dans les sondages effectués en rebord de plateau (fig. 1), les vestiges peu nombreux correspondent probablement à des pièces enfouies par les agents naturels (racines, rongeurs) depuis un niveau de sol aujourd'hui totalement détruit. De fait, tout le mobilier provient de l'éboulis de colluvionnement de la carrière. L'homogénéité de la série lithique est donc loin d'être acquise, même si l'ensemble du débitage et de l'outillage est caractéristique d'une seule période. L'absence de pièces incongrues dans le contexte du Mésolithique moyen n'exclut pas, toutefois, la possibilité de pièces plus anciennes ou plus récentes (fragment de pointe/ couteau à dos ?, fig. $7, n^{\circ}$ 6) qui pourraient intervenir dans la série.

6 L'attribution de la faune à la même période que l'industrie lithique est un autre point. Aucun élément strict ne vient rattacher l'os et le silex, les deux provenant de l'éboulis dans des colluvions qui semblent distinctes. Un essai de datation AMS a échoué, faute de collagène suffisant. Les espèces représentées, exclusivement sauvages, indiquent simplement une compatibilité mésolithique, sans certitude.

\section{Matières premières}

7 Un seul silex est présent. Il s'agit d'une variété strictement locale, affleurant actuellement dans les labours sur une large partie du Pays d'Auge. Sa couleur varie du beige au marron et du gris clair au gris foncé. Des points plus clairs de un à cinq millimètres sont dispersés dans la masse sans influer sur le débitage. Le cortex est fin et non roulé. La forme des rognons est assez tourmentée (cornes...). Le grain est fin et l'aspect plus ou moins satiné. Les inclusions grenues internes sont rares et de petite dimension, peu susceptibles de gêner le débitage. Cela surprend sur ce type de silex et témoigne d'une sélection drastique lors des ramassages. La taille moyenne de certains déchets de taille (fig. $2, \mathrm{n}^{\mathrm{o}} 1$ à 3 ; fig. $4, \mathrm{n}^{\mathrm{o}} 4$; fig. $7, \mathrm{n}^{\mathrm{o}} 3$ ) atteste du module des blocs disponibles (12 à $15 \mathrm{~cm}$ de longueur) tout à fait classique pour le secteur (Pays d'Auge). 
Figure 2 : Fumichon " Vallée de la Pâquine " : lames et courtes lames. Figure 2 : Fumichon "Valley of Pâquine": blades and short blades.
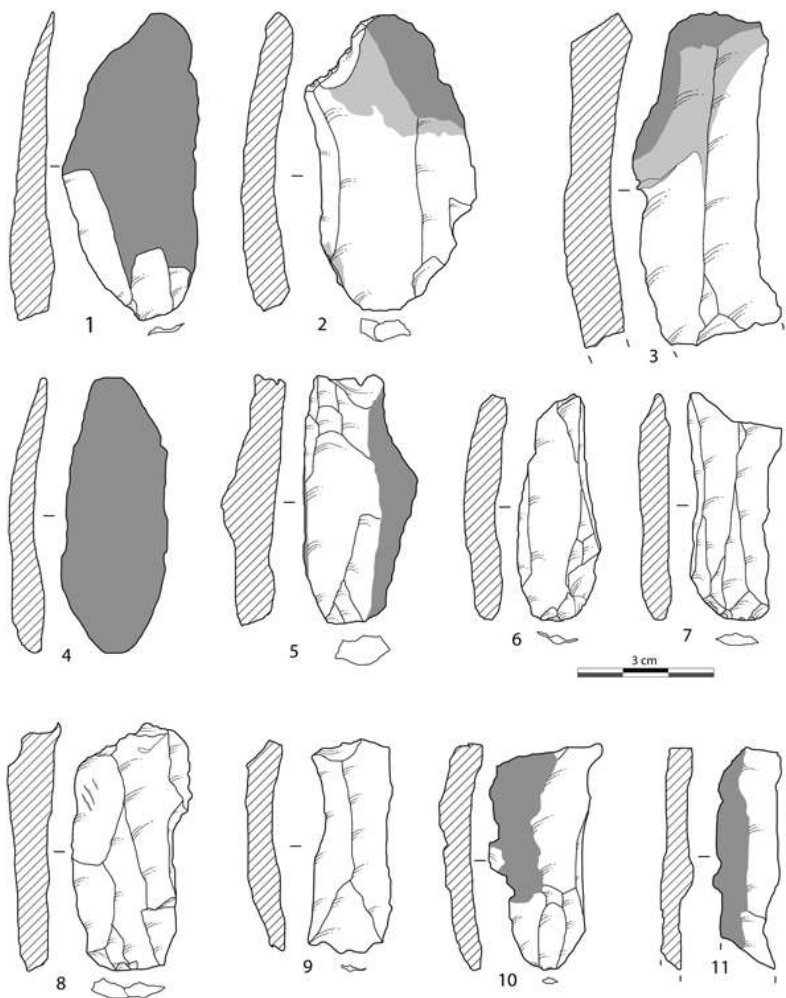

\section{Débitage}

La production est orientée préférentiellement vers l'obtention de produits laminolamellaires, que cela soit au niveau des déchets de taille ( $25 \%$ d'éclats, $60 \%$ de lames et lamelles) qu'au niveau des nucléus (3 nucléus à éclats seulement pour 29 à lamelles).

Les éclats d'initialisation sont très peu nombreux. Les pièces d'entame et de sousentame représentent moins de $6 \%$ des déchets supérieurs à $1,5 \mathrm{~cm}$ de longueur (88 pièces), ce qui témoigne dans le cas présent de deux facteurs. Le premier est l'importance des tests de matière première sur les lieux de ramassage. On note en effet une différence entre la bonne qualité du matériau dans la série et l'ensemble des blocs bruts que l'on peut observer localement en surface. La seconde raison de la présence discrète des phases d'initialisation est à l'image de la faible préparation du rognon préalablement au débitage laminaire/lamellaire, voire de l'absence de préparation pour les rognons les mieux adaptés. Sans être très fréquentes, quelques «lames et courtes lames » entièrement corticales (fig. $2, \mathrm{n}^{\circ} 1$ et 4 ) témoignent d'une entrée dans la phase de plein débitage lamino-lamellaire sitôt obtenu un plan de frappe approprié (après fracturation en deux parties d'un rognon par exemple).

\section{La production lamino-lamellaire}

Elle est représentée par plusieurs produits et sous-produits, principaux supports de l'outillage. Les lames, courtes lames et lamelles en sont les témoins essentiels; les éclats 
laminaires, éclats à enlèvements lamellaires, pièces à crête, pièces d'avivage et nucléus, les témoins indirects.

Les lames (83 pièces ; 5,6 \% de la série hors esquilles) sont des produits robustes et assez courts, généralement peu réguliers (fig. $3, \mathrm{n}^{\circ} 1$ à 3 ). Peu utilisées comme supports d'outils, ces pièces sont peu produites (fig. $7, \mathrm{n}^{\circ} 1$ à 3 ), peut-être à cause de la longueur limitée (probablement un maximum de $15 \mathrm{~cm}$ ) des blocs débités. Elles sont généralement à deux pans et épaisses. Leurs bords sont sinueux. Elles s'apparentent davantage aux lames du style de Rouffignac (Rozoy, 1978a) qu'à celles du style de Coincy. La préparation au débitage est très limitée: parfois, l'arête principale a été éliminée par esquillement. L'abrasion n'est jamais utilisée. Les talons sont larges et très épais. Les bulbes sont un peu proéminents et triangulaires, suggérant l'emploi de la percussion directe dure, tangentielle (fig. $3, n^{\circ} 1$ ) ou non (fig. $3, n^{\circ} 2$ ). L'épaisseur de ces pièces, leur faible régularité et le caractère entièrement cortical de certaines suggèrent le caractère aléatoire de leur production, très différente de celle des lamelles et courtes lames. Leur usage comme support d'outil allongé massif est toutefois incontestable (fig. $7, \mathrm{n}^{\circ} 1$ à 3 ).

Figure 3 : Fumichon «Vallée de la Pâquine » : courtes lames et lamelles. Figure 3 : Fumichon "Valley of Pâquine": short blades and bladelets.
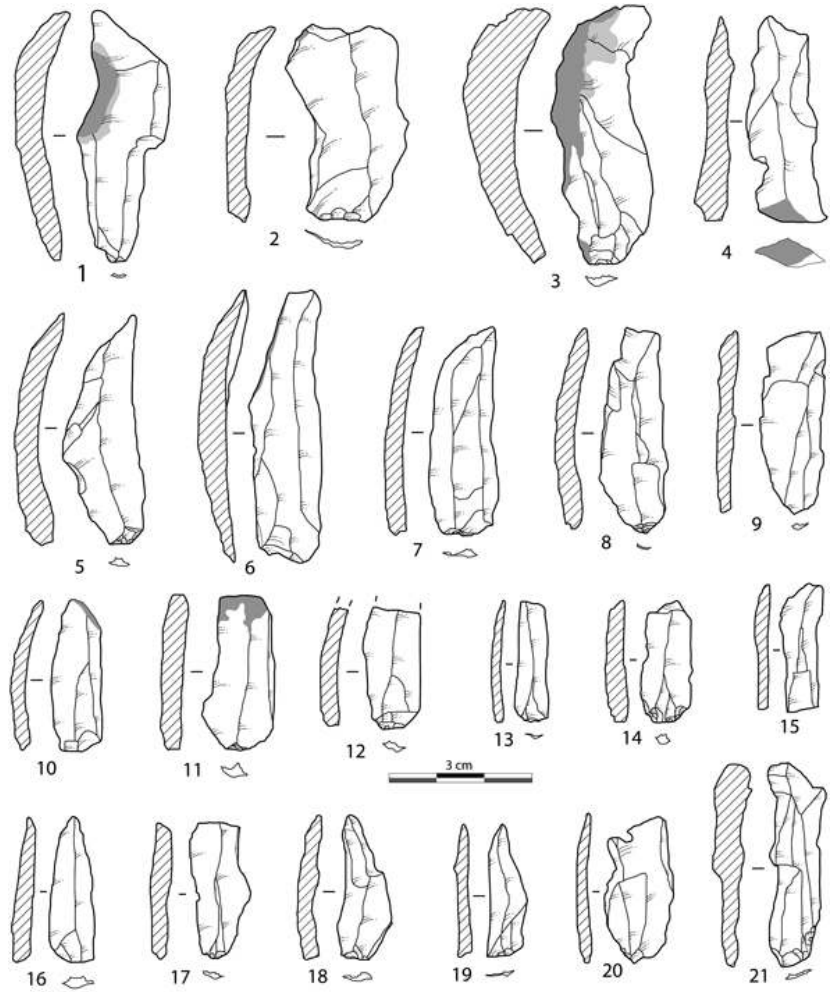

Les courtes lames ( 88 pièces; $6 \%$ de la série hors esquilles) sont classées dans cette catégorie à partir de leurs caractéristiques morpho-métriques (fig. $3, \mathrm{n}^{\circ} 4$ à 11 ; fig. 4 , $\mathrm{n}^{\circ} 1$ à 4 ). Elles sont en général trop courtes pour être considérées comme des lames $(\mathrm{L}<5 \mathrm{~cm}$ ) et trop larges ou trop épaisses pour être des lamelles (épaisseur $>5 \mathrm{~mm}$, inaptitude théorique à la réalisation d'armatures). En dehors de quelques pièces entièrement corticales (fig. $\left.3, n^{\circ} 4\right)$, les autres se partagent entre produits à deux et à trois pans. Les bords sont sinueux et l'épaisseur importante (6 à $8 \mathrm{~mm}$ en moyenne). La préparation au débitage est limitée. Les talons sont divers: certains larges et épais 
suggèrent l'emploi de la percussion directe rentrante. La plupart, larges et minces (voire punctiformes) suggèrent une percussion tangentielle. Les bulbes de percussion présentent un impact marqué "triangulaire »; ils sont très peu bombés avec une ou deux lancettes plus marquées et sans lèvre. L'hypothèse d'emploi du percuteur de pierre tendre est privilégiée sur le bois de cerf. Un seul fragment de galet allongé en grès tendre bouchardé a été découvert; il est tout à fait compatible avec le type d'impact identifié sur les courtes lames et les lamelles.

Les lamelles correspondent à l'objectif principal de la production et au support le plus recherché, non seulement pour la réalisation des armatures, mais aussi pour un nombre conséquent d'outils du fonds commun (lamelles retouchées et tronquées). Le choix des supports les plus réguliers pour la réalisation des armatures oblige par ailleurs à une production importante, la plupart des produits étant peu réguliers. 478 lamelles entières ou fragmentées ont été identifiées $(32,2 \%$ de la série hors esquilles, fig. $4, \mathrm{n}^{\circ} 5$ à 21 ). Ces produits présentent des modules divers, à l'image de leur utilisation également diversifiée parmi l'outillage. Les modules varient de pièces à la limite de l'esquille ( $2 \mathrm{~cm}$ de longueur pour 0,5 de largeur et 0,2 d'épaisseur, fig. $4, \mathrm{n}^{\circ} 13$ ), jusqu'aux lamelles de plus de $5 \mathrm{~cm}$ de longueur, supports des lamelles retouchées et tronquées. Les lamelles se partagent entre pièces à deux pans, dominantes dans l'ensemble, et celles à trois pans, qui sont souvent plus grandes. Elles sont d'épaisseur régulière et les profils sont droits ou très légèrement arqués. Leurs bords sont dans l'ensemble assez sinueux. Les plus régulières, bien rectilignes (fig. $4, \mathrm{n}^{\circ} 11$ à 13 ) sont minoritaires. La préparation de l'extraction consiste, comme pour les courtes lames, à une abrasion limitée. Les talons souvent minces ou punctiformes s'élargissent parfois, témoignant d'une régularité moyenne du débitage qui génère des accidents de taille (cf. éclats laminaires et éclats à enlèvements lamellaires). Les bulbes sont identiques à ceux des courtes lames: très peu bombés, sans lèvre et marqués par une ou deux lancettes. Ces caractéristiques renvoient de manière probable à la production directe tangentielle au percuteur (de pierre) tendre. Leur production s'inscrit dans la suite de celle des courtes lames et peut être rattachée au style de Coincy (Rozoy, 1978a). En dépit de l'apparente profusion des matières premières et du rejet de nucléus encore largement exploitables (fig. $4, \mathrm{n}^{\circ} 3,4$ ), la production lamellaire est généralement poursuivie jusqu'à l'extraction d'esquilles lamellaires $(<2 \mathrm{~cm}$, très minces), non exploitables pour la réalisation d'armatures: ces dernières sont en effet rarement pygmées $(<18 \mathrm{~mm})$ et jamais hyper-pygmées $(<12 \mathrm{~mm})$. 
Figure 4 : Fumichon "Vallée de la Pâquine » : nucléus. Figure 4 : Fumichon "Valley of Pâquine": nucléus.

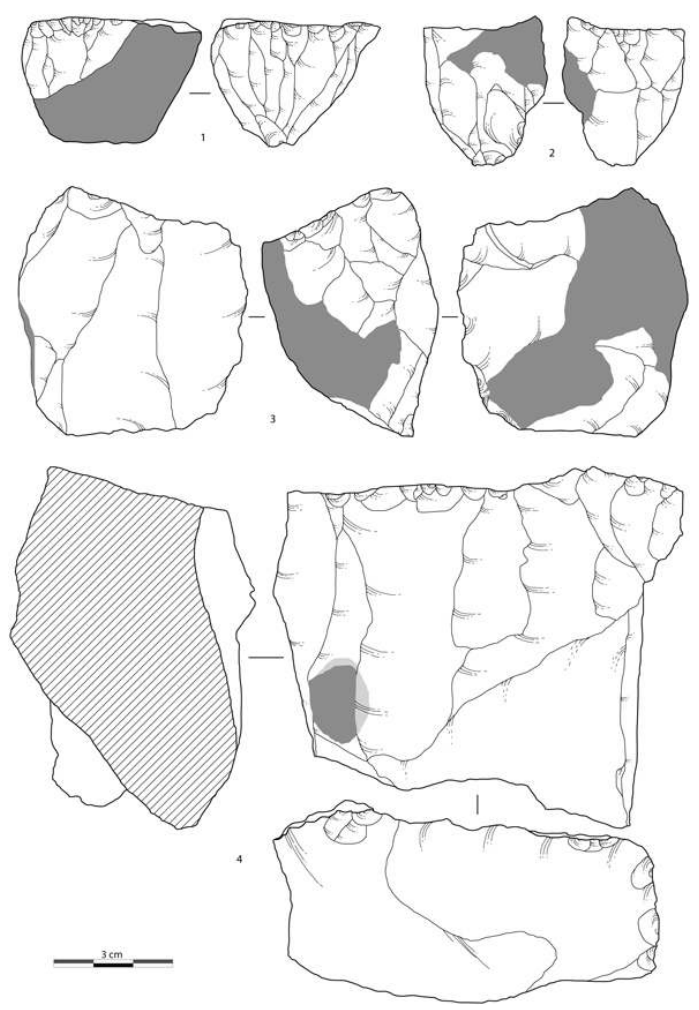

Plusieurs autres sous-produits appartiennent à la chaîne de production laminolamellaire. Même si ce ne sont pas les supports recherchés en premier lieu, certains ont fait l'objet d'une retouche et/ou d'une utilisation (éclats laminaires). D'autres catégories au contraire ne font l'objet d'aucune utilisation visible (éclats d'avivage, nucléus).

éclats laminaires et les éclats à enlèvements lamellaires sont les sous-produits de la chaîne de production lamellaire les plus communs (respectivement 186 et 23 pièces, soit 12,6 et $1,6 \%$ de la série hors esquilles). À quelques exceptions près, il semble qu'il s'agisse d'accidents de taille, très fréquents dans la production du style de Coincy (Rozoy, 1978a et b; Ghesquière et al., 2000). Les premiers sont des produits laminolamellaires trop courts; les seconds sont des produits lamellaires trop larges. Si la méthode de débitage semble identique à celle des autres produits "réussis » (percussion tangentielle à la pierre tendre probable...), l'impact a été porté nettement trop en arrière du bord de frappe et/ou porté de manière trop rentrante. Une mauvaise angulation du plan de frappe ou un geste mal maîtrisé expliquent aisément la multiplication de ce type d'accident qui ne semble jamais avoir entraîné l'abandon de nucléus (absence de nucléus couverts de négatifs de rebroussés).

Quatorze pièces à crête ont été mises en évidence ( $1 \%$ de la série). Elles sont réalisées sur éclat ou sur lame/lamelle. En aucun cas il ne s'agit de crête primaire de mise en forme du nucléus, mais de produits secondaires intervenant au cours du débitage.

Treize pièces d'avivage de plan de frappe ont été observées. Rarement sous forme de tablettes totales, elles sont destinées à rétablir une angulation appropriée au plan de frappe. Cette méthode semble peu utilisée à Fumichon; la percussion tangentielle semble, en règle générale, avoir peu recours à elle (Ghesquière et al., 2000). Dans une 
production du style de Coincy, les tailleurs privilégient un changement d'orientation des nucléus (nucléus croisés ou prismatiques).

Enfin, les derniers témoins de production de supports allongés sont 210 esquilles lamellaires (dont $5 \%$ présentent une surface corticale). Ces pièces témoignent pour une part d'accidents de taille (pièces trop minces ou trop courtes) et, pour une autre part, d'une prolongation du débitage au-delà du « raisonnable ».

Les nucléus présentant des négatifs d'enlèvements lamino-lamellaires sont au nombre de trente-six. La grande majorité est unipolaire (22 pièces; fig. 4). Les tables sont généralement étendues aux flancs du nucléus, parfois également au dos (3 nucléus pyramidaux). Leur rejet intervient généralement lorsque la hauteur n'atteint plus les $45 \mathrm{~mm}$ de hauteur. La plupart de ces pièces correspond à des nucléus sur éclat ; le plan de frappe est installé sur la surface de fracturation des rognons en deux parties exploitables.

\section{Le débitage d'éclats}

Une production d'éclats a été mise en évidence à partir de 369 déchets de taille ( $25 \%$ de la série). Un faible nombre de nucléus correspond à cette production (3 pièces). Les produits présentent des morphologies très diverses (fig. 6, $\mathrm{n}^{\circ} 1,4,6$ à 9,11 ). Aucun ne semble s'inscrire dans une chaîne de production lamino-lamellaire. Ce sont des pièces assez grandes : autour de $45 \mathrm{~mm}$ de longueur. Leur usage comme support d'outil est important (grattoirs et éclats retouchés/denticulés/à coche en particulier, soit un tiers de l'outillage). Leur production s'effectue à partir de nucléus multipolaires globuleux, sans préparation spécifique préalable. La production se prolonge, comme pour les lamelles, par l'extraction de pièces de petite taille, peu ou pas utilisées comme support d'outil. Les talons des éclats sont larges et épais. Les bulbes sont triangulaires avec des lancettes, suggérant une production au percuteur dur, en percussion directe rentrante. Aucun percuteur globuleux susceptible de correspondre à cette production n'a été identifié dans la série.

Le reste de la série est composé de pièces d'initialisation, de cassons, de pièces éclatées par le feu et d'esquilles.

- Les pièces d'initialisation ( 86 pièces, soit $5,7 \%$ de la série hors esquilles) sont larges et épaisses, généralement de grand module, utilisées pour définir un plan de frappe et créer un flanc. D'une manière générale, le faible nombre de pièces d'initialisation est un argument en faveur de l'hypothèse de nombreux tests du silex sur le lieu de ramassage (voir ci-dessus).

- Les cassons sont rares ( 5 pièces, soit $0,3 \%$ ). Ce pourcentage extrêmement faible indique avant tout l'absence de fissures internes des rognons de silex, reflet encore une fois de l'attention portée à la qualité de la matière première.

- Les débris brûlés sont au nombre de 51. Il s'agit de déchets de taille qui ont éclaté dans le feu, ce qui rend inidentifiable leur morphologie d'origine. Ils s'ajoutent aux soixante déchets de toutes catégories (cf. tableau fig. 5) qui ont subi l'action du feu sans avoir totalement éclaté. Sur l'ensemble, ces vestiges représentent 7,5\% de la série (esquilles non comprises), ce qui s'accorde avec les séries qualifiées de domestiques (Rozoy, 1978b ; Ghesquière et al., 2000).

- Le reste de l'assemblage est constitué de 210 esquilles lamellaires, 7 esquilles d'entame et 256 autres esquilles. Le nombre d'esquilles assez réduit (une pour trois déchets de taille) 
suggère un déficit général en pièces de petite dimension, qui s'explique par les conditions de ramassage de récolte (voir ci-dessus).

- Enfin, deux blocs testés et un bloc brut, en silex qui semble de qualité comparable au reste de la série, confirment l'abondance de silex aux alentours du site, tout comme quelques gros nucléus (fig. 4, $\mathrm{n}^{\circ} 4$ ).

Figure 5 : Fumichon "Vallée de la Pâquine » : tableau d'inventaire du mobilier lithique. Figure 5: Fumichon "Valley of Pâquine": inventory of the lithic assemblage.

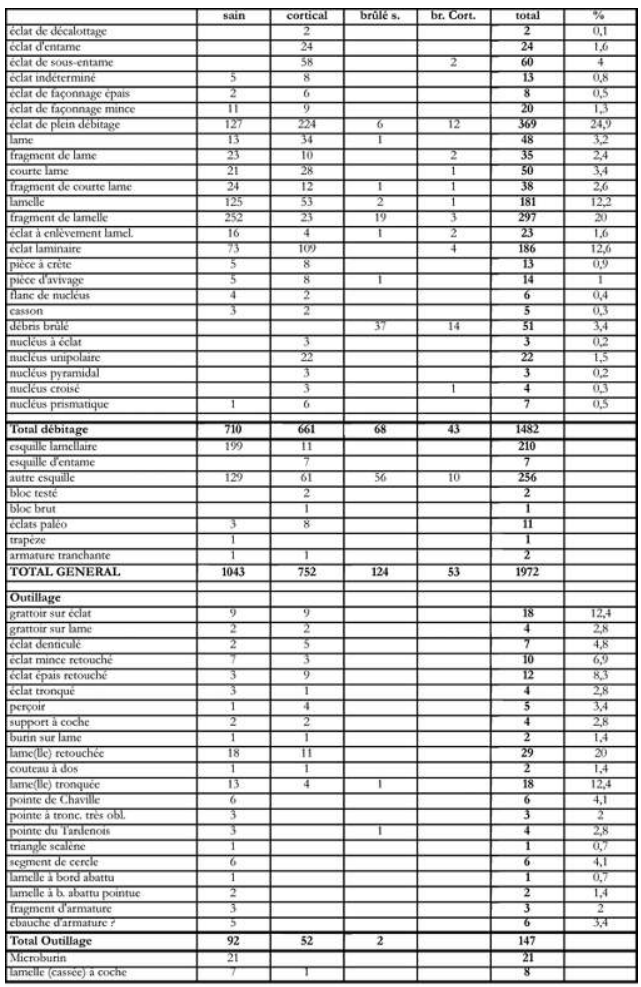

\section{Outillage}

Cent quarante-sept outils ont pu être isolés, soit pratiquement $10 \%$ de la série (esquilles non comprises). L'outillage se partage entre cent quinze outils du fond commun et trente-deux armatures. Ce rapport trois quarts/un quart s'exerce largement au profit de l'outillage du fonds commun. Par ailleurs, l'outillage sur éclats se compose de cinquante-six pièces et celui sur lames/lamelles de quatre-vingt-neuf. On note ainsi une surreprésentation du support éclat par rapport à sa représentation parmi les déchets de taille. Cela rejoint le déficit des armatures et des esquilles déjà évoqué.

\section{L'outillage du fonds commun}

La catégorie des grattoirs se compose de dix-huit pièces sur éclat et quatre sur lame (fig. $6, \mathrm{n}^{\circ} 1$ à 7). Pour les deux types de supports, ces outils se partagent équitablement entre pièces corticales ou non. Les grattoirs sur éclat sont représentés par dix pièces simples sur éclat, au front parfois étroit et faiblement convexe (fig. $6, n^{\circ} 2$ ), d'autres au front franchement convexe (fig. 6, $\mathrm{n}^{\circ} 7$ ) voire semi-circulaire (fig. $6, \mathrm{n}^{\circ} 5$ ). Les huit autres grattoirs sont denticulés, avec un front convexe au tracé gibbeux (fig. $6, \mathrm{n}^{\circ} 1$ et 
6). Les grattoirs sur lame sont tous des grattoirs raccourcis (fig. 6, $\mathrm{n}^{\circ} 3$ et 4). Le front est convexe, plus ou moins régulier et la retouche se prolonge, régulière et courte, sur les côtés.

Figure 6 : Fumichon «Vallée de la Pâquine ».

Figure 6: Fumichon "Valley of Pâquine".

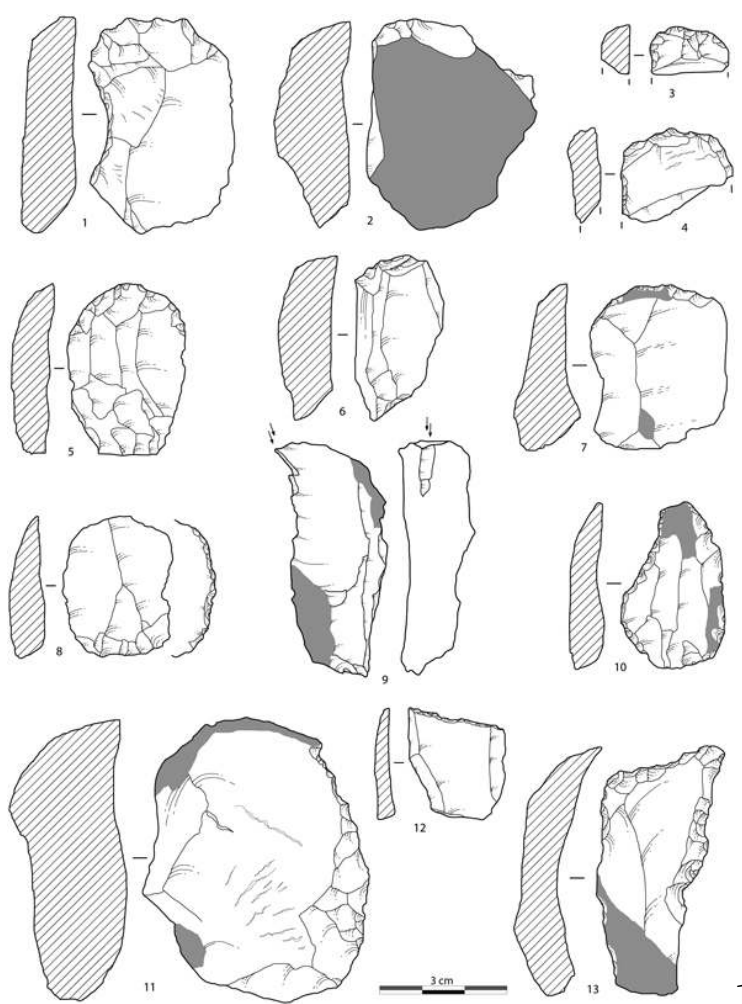
simples sur éclat ; 3,4 : grattoirs raccourcis ; $8,10,11,13$ : éclats retouchés ; 9 : burin sur cassure; 12 : éclat mince tronqué.

1: denticulated scraper; 2, 5, 6, 7: simple scrapers; 3 , 4: shortened scrapers; 8, 10, 11, 13: retouched flakes; 9: burin on break; 12: truncated flakes.

Les éclats retouchés et denticulés sont respectivement au nombre de vingt-deux et de sept (fig. $6, \mathrm{n}^{\circ} 8,10$ et 11 ). Ils sont réalisés à partir de supports de divers modules. La retouche est généralement régulière, étendue et se porte sur un des bords latéraux. Dans pratiquement tous les cas, elle semble avoir précédé l'usage.

Les éclats tronqués sont représentés par quatre pièces. Les supports sont variés et sont aussi bien des petites pièces (fig. $6, \mathrm{n}^{\circ} 12$ ) que des plus grandes et épaisses, toujours larges cependant. La retouche est rectiligne et abrupte, créant une troncature légèrement oblique par rapport à l'axe du débitage.

Cinq perçoirs (ou becs) sont présents. Ils sont exécutés sur support allongé, véritable lame (fig. $7, \mathrm{n}^{\circ} 4$ ) ou au moins éclat laminaire (fig. $6, \mathrm{n}^{\circ} 13$ ). La retouche est étendue, semi-abrupte à abrupte et exclusivement directe. Elle détermine une extrémité plus ou moins pointue, toujours robuste.

Les quatre pièces à coche sont des éclats et une lame (fig. 7, $\mathrm{n}^{\circ} 3$ ). La retouche profonde - directe dans trois cas et inverse dans un (celui sur lame) - détermine une coche unique régulière. 
Figure 7 : Fumichon « Vallée de la Pâquine ». Figure 7: Fumichon "Valley of Pâquine”.
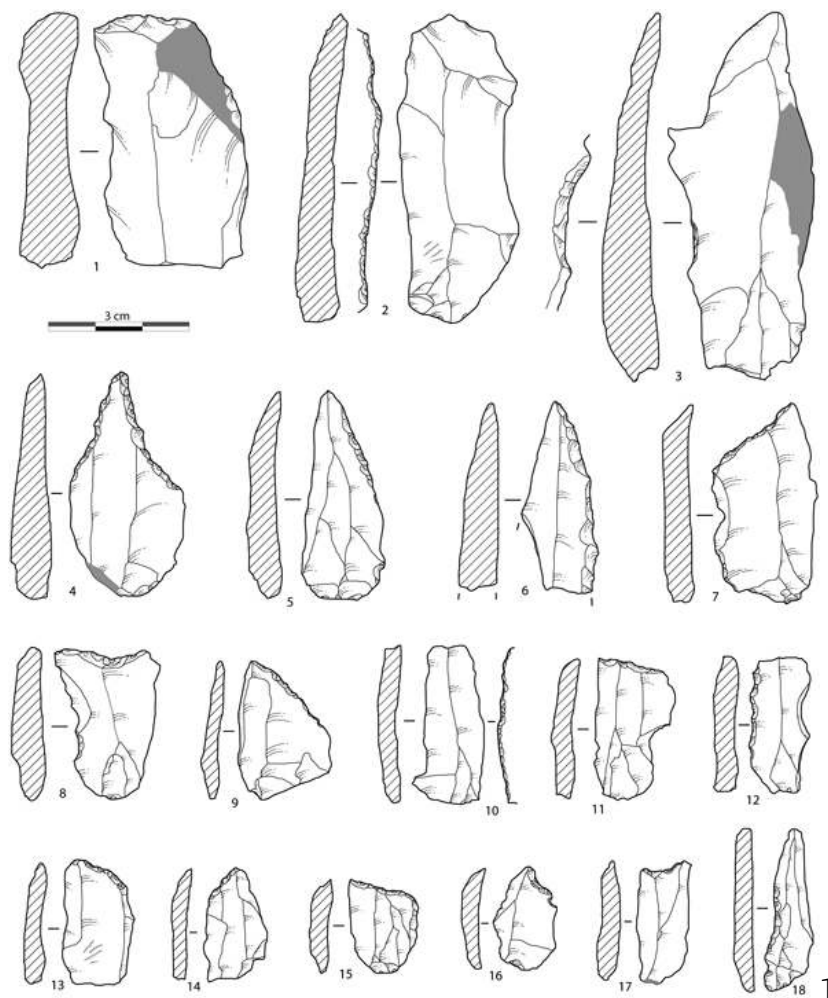

retouchées : 4 : perçoir sur lame $: 5,6$ : couteaux à dos courbe $: 7,8,9$ lamelles retouchées; 13, 14, 15, 16, 17 : lamelles tronquées.

1: scraper on blade; 2, 3: retouched blades; 4: perçoir on blade; 5, 6: knives with curved back; 7, 8, 9, 11: truncated blades, 10, 18: retouched bladelets; 13, 14, 15, 16, 17: truncated bladelets.

Deux burins ont été mis en évidence. Le premier est exécuté sur un éclat laminaire cortical, proche d'une lame du style de Rouffignac (fig.6, $n^{\circ} 9$ ). Les deux petits enlèvements ont porté à partir de l'extrémité distale de la pièce. Le second présente un enlèvement large débité à partir de la cassure d'une lame épaisse régulière.

Vingt-neuf lames ou lamelles retouchées ont été décomptées. Les supports de modules divers (toujours plus grands que ceux réservés aux armatures) portent une retouche plus ou moins régulière et continue directe ou inverse d'un de leurs bords (fig. $7, \mathrm{n}^{\circ} 2$, $10,12)$.

Deux couteaux à dos sont présents dans la série. Le premier présente une retouche semi-abrupte sur la moitié supérieure et une retouche semi-abrupte transverse de sa base (fig. $7, \mathrm{n}^{\circ}$ 5). Le second, incomplet, a une retouche abrupte courbe sur toute la partie du bord conservé (fig. $7, \mathrm{n}^{\circ}$ 6). Si les deux pièces se placent typologiquement dans la catégorie des couteaux à dos courbe, leurs faibles dimensions n'excluent pas qu'il s'agisse d'extrémités d'armes de jet.

31 Les lames et lamelles tronquées (18 pièces) concernent surtout des supports de petits modules (fig. 7, $\mathrm{n}^{\circ} 8,9,11,13,15$ à 17) et de quelques grandes pièces (fig. 7, $\mathrm{n}^{\circ} 1,7$ ). La retouche est directe abrupte, généralement rectiligne (un contre-exemple concave avec le $\mathrm{n}^{\circ} 8$ de la fig. 7). La troncature est plus ou moins oblique, se rapprochant parfois d'éventuelles ébauches d'armatures (fig. 7, nº 13 à 16). 


\section{Les armatures}

Vingt-six armatures (et six ébauches probables) ont été identifiées dans la série. On note qu'aucune d'entre elles ne présente de relique de zone corticale. La latéralisation à gauche est nettement dominante avec quatorze pièces contre deux à droite. Les autres pièces (segments symétriques) ne sont pas orientables.

Six pointes de Chaville sont présentes (fig. $8, \mathrm{n}^{\circ} 16$ à 21 ). Il s'agit de pointes courtes dont la retouche semi-abrupte à abrupte, rectiligne à légèrement convexe, n'occupe qu'une partie du bord, jamais jusqu'à la base. L'extrémité apicale est constituée d'un piquant trièdre non retouché. Certaines (toutes ?) semblent être des ébauches ou des ratées (fig. $8, \mathrm{n}^{\circ} 19$ et 21 ).

Figure 8 : Fumichon « Vallée de la Pâquine ".

Figure 8: Fumichon "Valley of Pâquine".
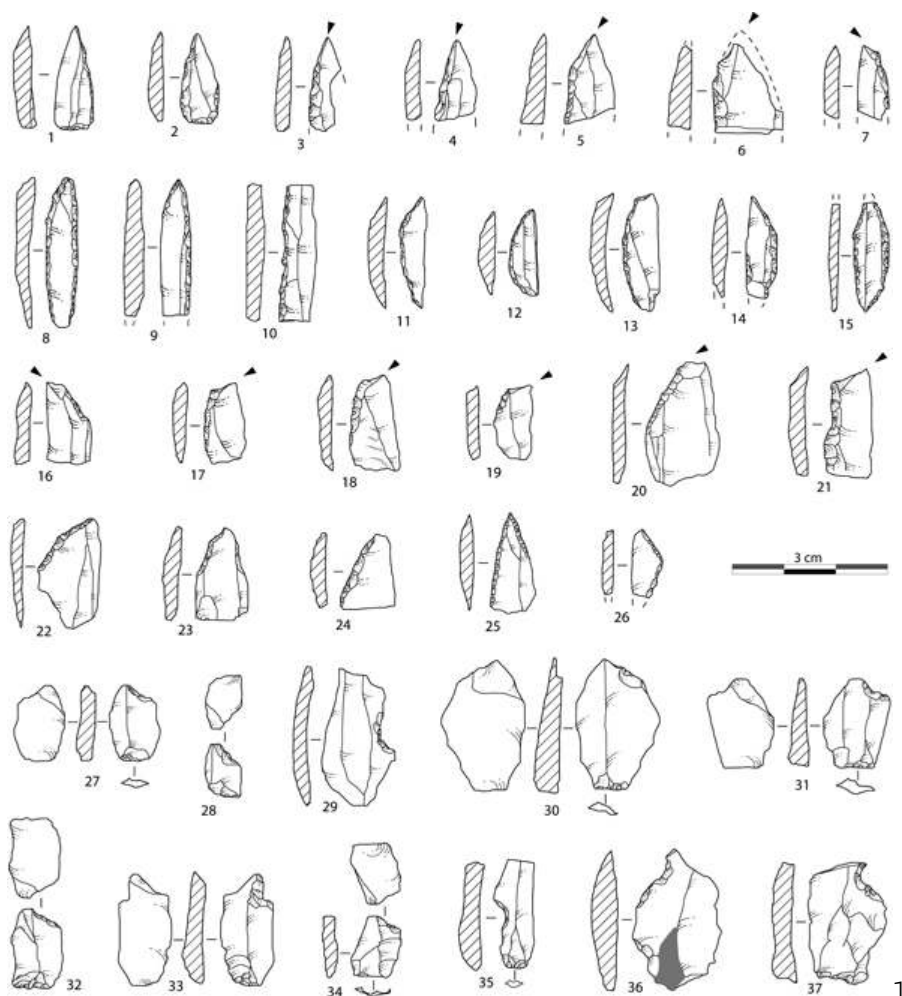

1,2 : pointes du Tardenois ; 3 4, 5, 6 : pointes du Tardenois possibles ; $7,11,12,13,14,15$ : segments ; 8,9 : lamelles étroites à bord abattu pointues, ou dards, ou segments atypiques ; 10 : lamelle étroite à bord abattu ; 16, 17, 18, 19, 20,21 : pointes de Chaville ou pièces inachevées à piquant trièdre ; $22,23,24,25$ : pointes à troncature très oblique ou ébauches ; 26 : triangle isocèle probable ; $27,28,30,31,32,33,34$ : micro-burins ; 29 , $35,36,37$ : lamelles à coche ou cassées dans la coche.

1, 2: points of Tardenois; 3, 4, 5, 6: possible points of Tardenois; 7, 11, 12, 13, 14, 15: segments, 8, 9: pointed backed bladelets; 10: backed bladelets; 16, 17, 18, 19, 20, 21: points of Chaville; 22, 23, 24, 25: points with oblique truncated; 26 : likely isosceles triangle; $27,28,30,31,32,33$, 34: microburins; 29, $35,36,37$ : small strips with coach or broken in coach.

Trois pointes à troncature très oblique ont été identifiées (fig. 8, $\mathrm{n}^{\circ} 22$ à 24). Leur morphologie les rapproche des pointes de Chaville (la retouche de la troncature n'atteint pas la base de la pièce) mais leur pointe ne semble pas réalisée par la technique du micro-burin. On note qu'elles ne sont guère éloignées de certaines pièces classées parmi les lamelles tronquées. 

28,30 à 34) et probablement huit lamelles à coche/cassées dans la coche (fig. 8, $\mathrm{n}^{\circ} 29,35$ à 37). Tous les modules de micro-burins sont présents, ce qui suppose leur usage pour former l'extrémité de certaines pointes (Chaville, Tardenois, voire segments) comme peut-être l'extrémité de certains outils du fonds commun (lamelles tronquées ?). La latéralisation à droite des micro-burins et lamelles à coche est largement dominante, ainsi que leur installation sur la partie proximale des lamelles.

\section{Discussion autour du mobilier : caractéristiques générales de la série}

43 Le silex débité est local, non fissuré et sans inclusions grenues. L'abandon de certains nucléus encore volumineux témoigne de l'abondance de la matière première, largement disponible actuellement en surface.

Le débitage est largement dominé par la production lamino-lamellaire. Les lames sont peu nombreuses en raison du module des rognons bruts, mais sont remplacées par des courtes lames technologiquement proches des lamelles. Ces dernières constituent un 
déchet de taille sur trois. Elles se partagent entre produits à deux ou trois pans. La préparation de l'extraction se limite dans le meilleur des cas à un esquillement limité des corniches. Les nucléus à lamelles sont dominés par les exemplaires unipolaires réalisés à partir de demi-rognons. Quelques nucléus pyramidaux, prismatiques ou croisés complètent la série. Enfin, un quart des déchets de taille correspond à des éclats, indispensables à la réalisation d'une partie de l'outillage du fonds commun (grattoirs, éclats retouchés...). Ce pourcentage important pourrait indiquer un déficit numérique en déchets lamino-lamellaires, transformés en outils et utilisés/perdus en dehors du site. Par ailleurs, la faible présence de nucléus à éclats pourrait indiquer le rejet hors du site de certains de ces nucléus, mais on ne peut exclure leur réutilisation pour une production lamellaire, moins dispendieuse en matière première.

L'outillage est dominé par les outils du fonds commun aux dépens des armatures (plus de deux pour une). Ce déficit en armatures témoigne des conditions de collecte de la série. L'outillage du fonds commun est dominé par les lamelles retouchées et tronquées (un tiers des outils) et, dans une moindre mesure, par les grattoirs et les éclats retouchés/denticulés. On note l'absence des éléments prismatiques, caractéristiques entre autres des contextes normands (Chancerel, 1983 ; Chancerel et Paulet-Locard, 1991 ; Souffi, 2004).

Les armatures sont dominées par les pointes, particulièrement les pointes larges (pointes de Chaville et/ou à troncature très oblique, morphologiquement très proches) et, dans une moindre mesure, par les pointes du Tardenois. Les éléments (possibles) de barbelures sont représentés par les segments; ces derniers, de grandes dimensions (parfois plus grands que les pointes), pourraient également être emmanchés en extrémité apicale des hampes de flèche. Un seul triangle (isocèle?) est présent. L'utilisation du procédé du micro-burin est observé sur une armature sur trois.

Enfin, le pourcentage de pièces ayant subi l'action du feu est de $7 \%$ des vestiges. Ce chiffre correspond globalement à ce que l'on considère comme normal pour une occupation domestique regroupée (Ghesquière et al., 2000).

\section{Comparaisons et attribution chrono-culturelle}

Le débitage lithique de Fumichon est comparable à de nombreux sites français du premier Mésolithique. Le style de Coincy, destiné à produire des supports lamellaires peu réguliers, est très largement mis à profit. Sans que ce soit le but recherché, on jugera de l'intérêt des bords sinueux comme ligne naturelle des armatures (pointe du Tardenois par exemple). Cette technique de débitage, très économe en silex et se passant parfois complètement de mise en forme du nucléus, est probablement celle qui représente le mieux la période mésolithique, généralement avare en matériau. Sa grande durée limite par contre les possibilités d'accroche chronologique précise (9500-9000 ? / 6400 cal. BC).

L'outillage permet des comparaisons plus précises, encore que le caractère ubiquiste de l'ensemble de ses composantes soit là aussi un obstacle à une détermination chronologique précise. L'outillage du fonds commun, dominé par les lames/lamelles retouchées, les éclats retouchés/denticulés et, dans une moindre mesure, les grattoirs, s'inscrit bien dans le premier Mésolithique de la moitié nord de la France, par comparaison avec des sites comme Argentan «Arma Maquettes» (Leroy, 1991), au 
niveau local, ou sur la Somme Beaulieu-les-Fontaines «La Haute Borne » (Fagnart et Roussel, 1981 ; Ducrocq, 2001) pour rester sur deux exemples peu éloignés.

L'outil qui aurait peut-être pu être déterminant, le grand prismatique en grès ou en silex, n'est pas présent à Fumichon; il est pourtant récurrent autour de la vallée de la Seine (Griselin, 2008 et Le Maux et Griselin, 2012).

Pour une approche, sinon chronologique, au moins comparative plus poussée, il faut prendre en compte la catégorie des armatures. Celles-ci sont dominées à Fumichon par les pointes à base naturelle, les pointes du Tardenois et les segments. Les comparaisons les plus évidentes et peut-être les plus pertinentes se tournent vers les séries picardes, comme Beaulieu-les-Fontaines (op. cit.), qui présentent les trois mêmes dominantes au début du Boréal. Le bassin de la Somme compte de nombreuses séries de la fin du préBoréal et du début du Boréal qui se caractérisent par l'abondance des segments et l'absence des triangles. C'est exactement la situation que l'on observe à Fumichon, avec un seul triangle, si tant est que l'on puisse considérer la série comme représentative. À Argentan, à cinquante kilomètres au sud-ouest du site, la proportion entre segments et triangles est encore favorable aux premiers, alors qu'elle est de deux triangles pour un segment encore plus au sud à Saint-Ellier-les-Bois (Collectif, 2010), à quatre-vingts kilomètres au sud-ouest de Fumichon. Plus au sud (Pays de la Loire), les segments deviennent anecdotiques, les triangles dominant tout le corpus (fig. 9 ; Michel, 2011), à quelques exceptions près comme le site de Saint-Romain-sur-Cher-loc 3 (Kildéa, 2008). Ces séries à segments sont mieux représentées en Île-de-France (Boinvilliers «Le Dentu »; Griselin, 2008) et dans l'Yonne, sur des sites comme Saint-Julien-du-Sault "Les Sablons» (Peretto et Thévenin, 1995) ou Saint-Privé "Les Quatre Arpents » (Huchet et Thévenin, 1995) associés à des pointes du Tardenois (voire de Beaugency), des pointes à base naturelle et des pointes de Sauveterre (voire de Chateaubriand). Cette mixité des assemblages, également observée à Alizay "Le Postel» (Marcigny, fouille en cours), de pointes de Sauveterre/segments/pointes du Tardenois, caractérise les gisements du sud de la Seine. À Fumichon, il semble que l'on soit proche des séries qui s'étendent de la Picardie au nord du département de l'Orne, où les segments laissent très peu de place aux triangles. Les pointes de Chaville, quant à elles, sans être très nombreuses à Fumichon, sont une des caractéristiques des sites de l'île-de-France et de ses marges, comme par exemple Saint-Julien-du-Sault «Les Sablons » (Yonne, Peretto et Thévenin, 1995) ou Chaville 3 (Rozoy, 1978b), se prolongeant en Normandie par le petit gisement de Valframbert "Le Moulin d'Aché » (Collectif, 2010) ou celui d'Acquigny « L'Onglais » (Souffi, 2004 et 2008). 
Figure 9 : Partage du nord-ouest de la France entre les deux grands complexes de barbelures, segmentiformes dans le nord-ouest, triangulaires isocèles dans le sud et le nord-est, et mixte " entre Seine et Loire ». Quelques sites de référence du pré-Boréal ou de la transition pré-Boréal/ Boréal sont également figurés.

Figure 9 : division of the northwest of France between two big complexes of segments in northwest, triangular isosceles in the South and the northeast, and mixed "between the Seine and the Loire". Some reference sites of the Preboreal / boreal Preboreal or the transition are also represented.

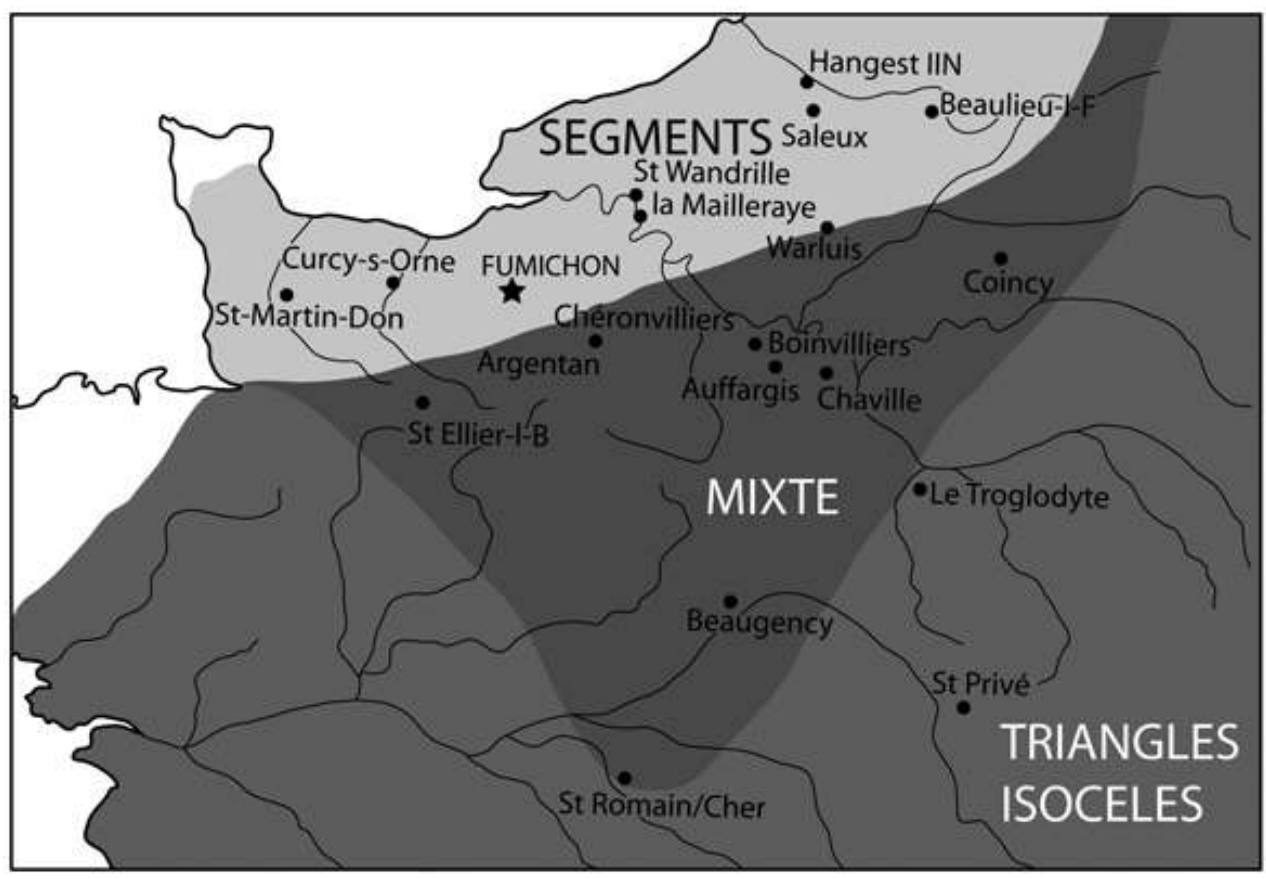

D'un point de vue chrono-culturel, les pointes de Chaville et les ensembles à segments sont d'ordinaire placés dans la seconde moitié du Mésolithique ancien et le premier tiers du Mésolithique moyen. Cette période un peu "fourre-tout» est celle où l'outillage mésolithique a vraiment pris ses marques (le débitage laminaire et les burins deviennent rares) et où les armatures du début du pré-Boréal (grandes pointes à troncature) et celles du Boréal (pointes du Tardenois) cohabitent. En revanche, cet épisode précède l'intervention des assemblages nordiques à feuilles de gui et lamelles étroites à bord abattu (RMS) du milieu du Boréal; entre les deux, semblent même s'inscrire des ensembles à triangles et pointes à base retouchée (Ducrocq, 2001). En Bretagne, dans des contextes culturels différents, c'est durant cette interface chronologique que l'on situe le Berthaume (Nicolas et Marchand, 2010; Blanchet et al., 2006), mais un Mésolithique "ancien " à triangles est également présent, comme à Kerjouanno (Dietsch, 1991). Grégor Marchand préfère ainsi parler de premier Mésolithique, du fait de la difficulté à séparer ce qui relève du Mésolithique ancien et ce qui relève du moyen.

Pour ce qui concerne les attributions en chronologie absolue, plusieurs séries comparables du nord-ouest de la France possèdent des datations assez précises. Celles d'Hangest-sur-Somme « Gravière II Nord » (Ducrocq, 2001), avec son cortège de pointes à base non retouchée, segments et pointes du Tardenois sont un peu larges $(8830 \pm 90$ BP et $8740 \pm 80$ BP ; Ducrocq, 2001). Plus précises sont les datations obtenues sur les séries de Saleux « niv. inf ». (8645 \pm 70 ; Fagnart et al., 2008) ou Warluis « site IIc » (9000 \pm 50 ; Ducrocq et al., 2008), qui renvoient tous deux à des séries à segments et pointes à base retouchée ou naturelle. Celle de Saint-Romain-sur-Cher «loc. $3 »$ (Kildéa, 2008), 
dont l'assemblage est comparable mais dont la situation géographique est plus éloignée, présente une datation presque identique $(8820 \pm 50)$.

\section{Les vestiges osseux (P. Auguste)}

Le matériel faunique, pauvre et fragmentaire, se compose de dix restes. Une datation radiocarbone a été tentée sur un des plus gros restes osseux de la série. Le collagène y était hélas trop faible pour permettre une mesure d'âge.

- demi-poulie distale non épiphysée de métatarsien III-IV ; jeune cerf,

- phalange proximale II ou IV ; sanglier ; marques de rognage par un petit carnivore,

- fragment de diaphyse de métatarsien III-IV ; chevreuil,

- fragment de diaphyse d'os long; lagomorphe ou petit carnivore,

- tête et fragment de diaphyse de fémur ; petit carnivore,

- fragment de diaphyse d'os long; présence d'une fracturation hélicoïdale, de stries de découpe, de marques de passage au feu,

- fragment d'os ; brûlé,

- trois fragments osseux.

Cette très petite série faunique se caractérise donc par la présence du cerf, du chevreuil, du sanglier et d'un petit carnivore. Deux pièces montrent une action anthropique liée à l'alimentation, le fragment de diaphyse témoignant probablement d'une récupération de la viande avant la fracturation de l'os frais pour en extraire la moelle. Cet os est par ailleurs brûlé, ainsi que le deuxième vestige.

L'association cerf-sanglier-chevreuil est tout à fait caractéristique d'une phase nettement tempérée, dans un milieu à dominante boisée. Elle apparaît donc tout à fait en cohérence avec le contexte archéologique d'où elle provient, en l'occurrence une occupation mésolithique, ce tryptique faunique étant en effet très commun durant cette période (souvent accompagnée par ailleurs de l'aurochs).

\section{Conclusion}

La série de Fumichon offre une rare opportunité d'étude d'une série qui semble homogène $\mathrm{du}$ premier Mésolithique (fin $\mathrm{du}$ Mésolithique ancien ou plus vraisemblablement début du Mésolithique moyen). Le mobilier abondant permet une appréciation correcte de la série, avec 1500 pièces, esquilles non comprises, et 147 outils. Seul le nombre d'armatures est un peu limité, avec 32 pièces. Ce chiffre assez faible peut être rapproché de celui, limité lui aussi, des esquilles, pour lesquelles des raisons taphonomiques de conservation du gisement ont été évoquées, ainsi que par l'absence d'un tamisage général des sédiments. Malgré ces freins à une bonne lecture statistique et interprétative de l'outillage, la série offre plusieurs témoins pour une compréhension chrono-culturelle du site. La découverte de quelques ossements animaux permet d'observer, pour la première fois en Basse-Normandie, une association éventuelle de faune et d'industrie mésolithique, mais l'agrégation des deux types de mobilier est loin d'être acquise. 


\section{BIBLIOGRAPHIE}

BLANCHET S., KAYSER O., MARCHAND G. et YVEN E., 2006 - « Le Mésolithique moyen en Finistère : de nouvelles datations pour le groupe de Bertheaume ", Bulletin de la Société préhistorique française, t. $103, \mathrm{n}^{\circ} 3$, p. 507 à 518.

CHANCEREL A., 1983 - « La série mésolithique de Saint-Wandrille-Rançon (Seine-Maritime) », Bulletin de la Société préhistorique française, t. 80, p. 335-348, 11 fig.

CHANCEREL A. et PAULET-LOCARD M.-A., 1991 - « Le Mésolithique en Normandie », Mésolithique et néolithisation en France et dans les régions limitrophes, Actes du $113^{e}$ Congrès national des sociétés savantes, Strasbourg 1988, Paris, 1991, p. 213 à 230.

COLLECTIF, 2010 - Bilan de la recherché archéologique en Basse-Normandie, 1984-2010 - Du Paléolithique à la fin de l'âge du Fer, SRA, MCC, vol. 1, Préhistoire-Protohistoire, 2010, 176 p.

DIETSCH M.-F., 1991 - Le Mésolithique ancien et moyen dans le Nord de la Bretagne, Mémoire de maîtrise, université Paris X., 209 p.

DUCRoCQ T., 2001 - Le Mésolithique du bassin de la Somme, Publications du CERP, nº 7, 2001, 255 p.

DUCROCQ T., 2008 - « Le gisement de Warluis (Oise) : approche préliminaire », Le début $d u$ Mésolithique en Europe du Nord-Ouest, Actes de la table ronde d'Amiens, 9 et 10 octobre 2004, Mémoire XLV de la SPF, 2008, p. 85 à 106.

FAGNART J.-P. et ROUSSEL M.-C. et J.-P., 1981 - « Le gisement mésolithique de la Haute Borne à Beaulieules-Fontaines (Oise) », Revue archéologique de l'Oise, $\mathrm{n}^{\circ} 24$.

FAGNART J.-P., COUDRET P. et SOUFFI B., 2008 - « Les occupations mésolithiques du gisement de Saleux ", Le début du Mésolithique en Europe du Nord-Ouest, Actes de la table ronde d'Amiens, 9 et 10 octobre 2004, Mémoire XLV de la SPF, 2008, p. 107 à 135.

GHESQUIERE E., LEFEVRE P., MARCIGNY C. et SOUFFI B., 2000 - Le Mésolithique moyen du Nord-Cotentin, BasseNormandie, France, BAR International Series, Oxford, $n^{\circ} 856,292$ p.

GHESQUIERE E., DEDOUIT J.-J. et THOMAS Y., 2006 - « Le Mésolithique ancien dans le Calvados, Archéologie et prospection en Basse-Normandie ", Mémoires de la Société des antiquaires de Normandie, t. XXXVIII, p. 101 à 113.

GRISELIN S., 2008 - « Le substrat mésolithique dans le nord du département des Yvelines : caractérisation des industries et des contraintes d'implantation », Le début du Mésolithique en Europe du Nord-Ouest, Actes de la table ronde d'Amiens, 9 et 10 octobre 2004, Mémoire XLV de la SPF, 2008, p. 183 à 194.

LE MAUX N. et GRISELIN S., 2012 - «L'exploitation des grès-quartzite au Mésolithique et au Néolithique en Île-de-France ", in Marchand G. et Querré G. (dir.), Roches et Sociétés de la Préhistoire, entre massifs cristallins et bassins sédimentaires, Presses universitaires de Rennes, p. 179 à 194.

HUCHET A. et THEVENIN A., 1995 - « Les occupations mésolithiques des Quatre Arpents à Saint-Privé (Yonne) », Épipaléolithique et Mésolithique du Sénonais et des régions voisines, Actes du Colloque de Passy, 20-21 novembre 1993, cahier nº 2, p. 87 à 96. 
KILDEA F., 2008 - « Les occupations de Mésolithique ancien et moyen de Saint-Romain-sur-Cher (Loir-et-Cher) », Le début du Mésolithique en Europe du Nord-Ouest, Actes de la table ronde d'Amiens, 9 et 10 octobre 2004, Mémoire XLV de la SPF, 2008, p. 153 à 168.

LEROY D., 1991 - «Le site mésolithique d'Arma-Maquette à Argentan, Orne », Revue archéologique de l'Ouest, t. 8, p. 25-46, 16 fig.

MICHEL S., 2011 - Les premiers groupes mésolithiques de la France atlantique : enquête sur l'industrie lithique, Université de Rennes 1, 2011, thèse inédite, 536 p.

NICOLAS E. et MARCHAND G., 2010 - « Pen Hoat Salaün - Une occupation du Mésolithique moyen (Pleuven, Finistère) », Rapport final d'opération, Inrap Grand-Ouest, septembre 2010, 247 p.

PERETTO D. et THEVENIN A., 1995 - « Le gisement épipaléolithique et mésolithique des Sablons à Saint-Julien-du-Sault (Yonne) », Épipaléolithique et Mésolithique du Sénonais et des régions voisines, Actes du Colloque de Passy, 20-21 novembre 1993, cahier nº 2, p. 63 à 86.

PHILIBERT S., 2002 - Les derniers « sauvages » - Territoires économiques et systèmes techno-fonctionnels mésolithiques, BAR International Series 1069, 2002, 193 p.

ROZOY J.-G., 1978a - « Typologie de l’Épipaléolithique (Mésolithique franco-belge) », Bulletin de la Société archéologique champenoise, $\mathrm{n}^{\circ}$ spécial, juillet 1978, 120 p.

ROZOY J.-G., 1978b - « Les derniers chasseurs », Bulletin de la Société archéologique champenoise, nº spécial, juin 1978, 3 vol., 1256 p., 259 fig.

SOUFFI B., 2004 - Le Mésolithique en Haute-Normandie (France) - L'exemple du site d'Acquigny "L'Onglais » (Eure) et sa contribution à l'étude des gisements mésolithiques de plein air, BAR International Series 1307, 2004, $208 \mathrm{p}$.

SOUFFI B., 2008 - « Le Mésolithique en Haute-Normandie : taphonomie et interprétation chronoculturelle », Le début du Mésolithique en Europe du Nord-Ouest, Actes de la table ronde d'Amiens, 9 et 10 octobre 2004, Mémoire XLV de la SPF, 2008, p. 135 à 152.

\section{RÉSUMÉS}

Des ramassages et des sondages ponctuels sur la commune de Fumichon, dans les environs de Lisieux (Calvados) a permis l'observation d'une importante série lithique attribuable au Premier Mésolithique. Le corpus d'armatures est dominé par les segments, les pointes du Tardenois et les pointes de Chaville. L'assemblage caractéristique offre l'opportunité d'observer une série à segments et sans triangle au sud de la Seine. Quelques restes osseux, sans liens stratigraphiques stricts avec le mobilier lithique, relèvent d'une faune sauvage tempérée.

\section{INDEX}

Keywords : The ancient/middle Mesolithic, the points of Tardenois, the points of Chaville, segments, fauna

Mots-clés : pointes du Tardenois, pointes de Chaville, segments, Mésolithique, faune 


\section{AUTEURS}

\section{EMMANUEL GHESQUIÈRE}

Inrap, UMR 6566-CReAAH, 4 bd de l'Europe 14540 BOURGUEBUS, emmanuel.ghesquiere@inrap.fr.

PATRICK AUGUSTE

CNRS, Université de Lille.

JEAN-LUC PIEL-DESRUISSEAUX

14100 MAROLLES, jlcpieldx@hotmail.fr 